\title{
Changes in Sterol Lipids of Axis and Root of Germinating Soybeans
}

\author{
Hiromi Yoshida and Gorō KaJimoto \\ Faculty of Nutrition, Kobe-Gakuin University, Tarumi-ku, Kobe, Japan
}

Received September 3, 1979

\begin{abstract}
Soybean seedlings were grown at $28^{\circ} \mathrm{C}$ in the dark or the light for 12 days, and four classes of sterol lipids, sterol esters (SE), free sterols (St), acylated steryl glycosides (ASG) and steryl glycosides (SG), were isolated from the axis and root, respectively. Each sterol lipid (SE, ASG and SG) obtained was hydrolyzed and then divided into sterol, fatty acid and/or sugar fractions. The hydrolysates and St were analyzed mainly by gas-liquid chromatography (GLC).

During germination, the amounts of ASG markedly increased, especially in the lightgrown seedlings, whereas SE maintained almost constant values. The compositions and changing patterns of the sterol in sterol lipids were similar between the dark-grown and light-grown seedlings, and among epicotyl, hypocotyl and root. The changing patterns of fatty acid composition differed between SE and ASG. SE was found to have high proportions of unsaturated fatty acids, whereas ASG had high proportions of saturated fatty acids. In general, the changes in constituents of sterol lipids were more marked in the light-grown seedlings than in the dark-grown ones.
\end{abstract}

Lipids are known to have several functions in seeds: as reserve energy source, e.g., oil seeds; as necessary components of membrane systems. ${ }^{1,2)}$ It is considered that sterol lipids contribute to the stability of membrane structure with glycolipids and phospholipids. With respect to sterol lipids of germinating seeds, a few investigations have been reported by Kemp, ${ }^{3}{ }^{3} \mathrm{H} \mathrm{Imer}^{4}$ and Katayama et al.," using maize shoots, barley embryo and soybean seedlings, respectively.

Although a lot of information has accumulated about these plant lipids in the last few years, their significance for the plant itself is poorly understood. In a previous paper, ${ }^{6}$ we reported on changes in lipid component and fatty acid composition of axis and root of germinating soybeans. Hence, it would be of interest to clarify the physiological function of sterol lipids in germinating soybean grown either in the dark or the light. This paper deals with the isolation of four classes of sterol lipids (SE, St, ASG and SG) from the axis and root of soybean seedlings and deals with the changes in the chemical compositions of the sterol, fatty acid and sugar constituent of them.

\section{MATERIALS AND METHODS}

Germination of seeds. The mature seeds used in this experiment were an early variety of soybean, "Okuhara," harvested in Hokkaido Prefecture in 1978. The seeds were germinated and then grown for 12 days by the method described in a previous report. ${ }^{7)}$

Disstection of tissues. After 2, 4, 6, 8 and 12 day immersion in a growth chamber, the seedlings were separated into axis, root and other tissues with a microspatula. The axis used in this experiment was further separated into hypocotyl and epicotyl (containing young leaves). The other tissues contained hull and cotyledons.

Extraction and analysis of lipids. The dried samples as described in a previous report ${ }^{\mathrm{b})}$ were extracted three times with chloroform-methanol $(1: 1$ and $2: 1$, by vol.) in a Waring Blender. The combined extracts of total lipids obtained from each tissue were purified by a modification of the method of Folch et al., ${ }^{87}$ and then dried over anhydrous $\mathrm{Na}_{2} \mathrm{SO}_{4}$. The solvents were removed from the filtrates with a rotary evaporator under nitrogen at $30 \sim 35^{\circ} \mathrm{C}$. The lipids obtained were fractionated on Florisil column chromatography by the same methods as in the previous report.? The sterol lipids fractionated by rechromatography were checked by thin-layer chromatography (TLC). SE and St were isolated by one-dimensional TLC on silica gel plates, and ASG and SG by a double development technique on the plates, respectively. The solvent system used was $n$-hexane-benzene $(60: 40$ or $40: 60$, 
by vol.) for SE and St. Benzene-methanol $(98: 2$, by vol.) for the first development, and chloroformmethanol-acetic acid-water $(90: 8: 1: 1$, by vol.) for the second, were used for the separation of ASG and SG. Each of the sterol lipids was visualized with iodine and isolated by preparative TLC. Each sterol lipid (SE, ASG and SG) obtained was hydrolyzed with anhydrous $5 \% \mathrm{HCl}$ in methanol ${ }^{2)}$ and then separately divided into sterol, fatty acid and/or sugar fractions. The hydrolysates and St were analyzed mainly by GLC.

Gas chromatographic analysis. GLC analysis was performed on a gas chromatograph (Shimadzu GC7AG, Ltd., Kyoto) equipped with a hydrogen flame ionization detector. Fatty acid methyl esters, trimethylsilyl ether derivatives of sterols and sugars were prepared by the same technique as described in a previous report, ${ }^{7)}$ respectively. Fatty acid methyl esters were chromatographed on a glass column packed with $15 \%$ DEGS on Celite $545(0.3 \times 210 \mathrm{~cm})$. Trimethylsilyl ether derivatives of sterols and sugars were chromatographed on a glass column packed with $1.5 \%$ SE-30 (a silicone gum) on Chromosorb W-AW $(0.3 \times 210 \mathrm{~cm})$. The quantities of the sterols and sugars were calculated from the ratios to the internal standards, squalane and xylitol, respectively. All the other GLC conditions and parameters were the same as described previously.?

\section{RESULTS}

\section{Changes in four classes of sterol lipids in each tissue}

The results are based on 100-seed lots germinated for zero to 12 days. The changes in the sterol contents of the four classes of sterol lipids in epicotyl, hypocotyl and root of soybean seedlings are shown in Figs. 1, 2 and 3 , respectively. The results represent sterol content of sterol lipids. The total sterol contents in the three tissues gradually increased with the progress of soybean seedlings. The amounts of total sterols were more marked in the light-grown seedlings than in the dark-grown ones, especially in epicotyl and hypocotyl in the later stages of germination.

Under the two conditions tested, the major components in the three tissues were St during germination and also ASG in the final stage of germination, the minor one being SE. With the progress of soybean seedlings, the ASG in each tissue markedly increased

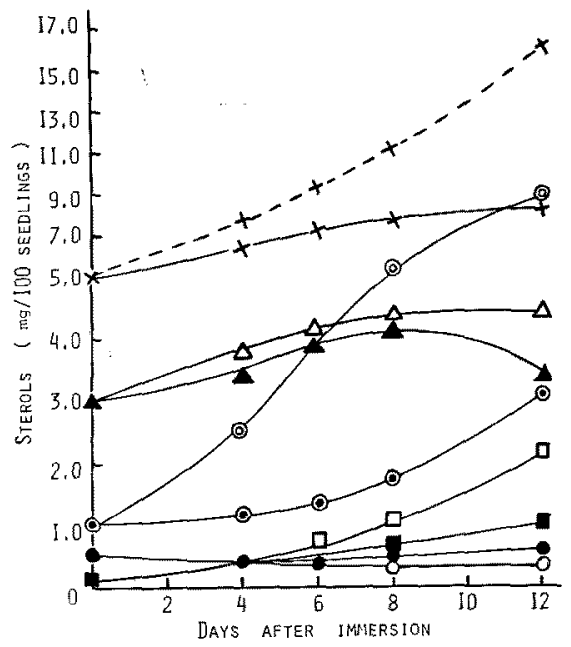

FIG. 1. Changes in Sterol Contents of Four Classes of Sterol Lipids in Epicotyl of Soybean Seedlings during Germination.

St: $\wedge$, dark; $\triangle$, light. SE: $\bullet$, dark; $O$, light. ASG: $\odot$, dark; $\odot$, light. SG: $\mathbf{E}$, dark; $\square$, light. Total sterols: $-\times-$, dark; $--\times-\cdots$, light. Abbreviations: see synopsis,

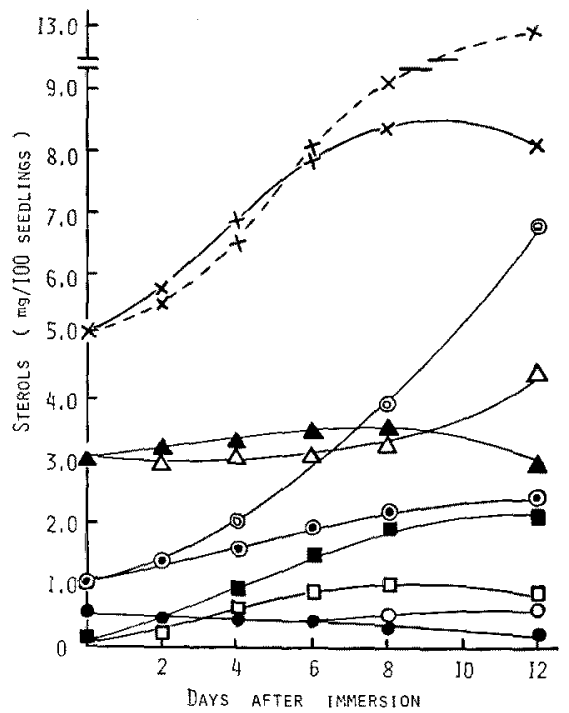

FIG. 2. Changes in Sterol Contents of Four Classes of Sterol Lipids in Hypocotyl of Soybean Seedlings during Germination.

The symbols are the same as in Fig. 1.

from the 4 th day after immersion and then became predominant in the light-grown seedlings in the later stages. Perphaps owing to the greening that occurred when soybean 


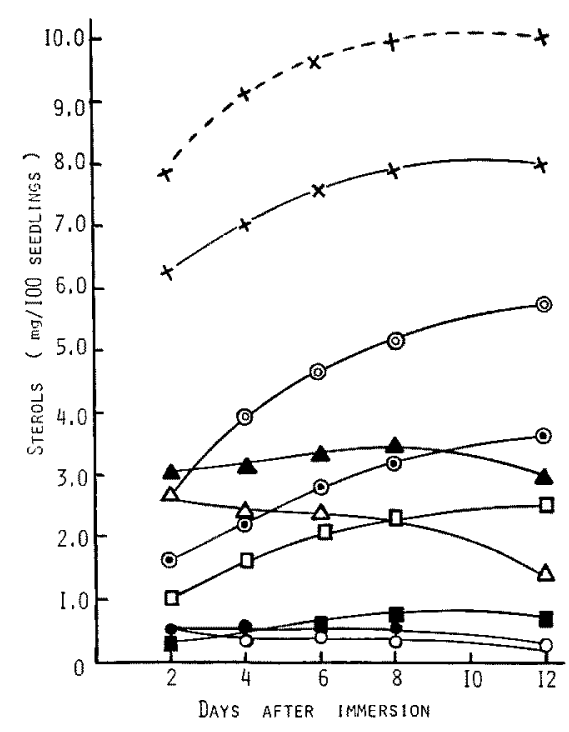

FIG. 3. Changes in Sterol Contents of Four Classes of Sterol Lipids in Root of Soybean Seedlings during Germination.

The symbols are the same as in Fig. 1.

seedlings were under the light, the photosynthetic tissues developed in the light-grown seedlings, especially in epicotyl containing young leaves. The SG in the three tissues increased with the progress of soybean seedlings. The changes in the St and SG were more marked in the light-grown seedlings than in the dark-grown ones, with the exception of hypocotyl. During germination of soybean seeds, the changes in SE were not observed as clearly as those in the other sterol lipids. The changing patterns of four classes of sterol lipids in each tissue were similar between the dark-grown and light-grown seedlings, with a few exceptions. The results markedly differ from the changes in them which were observed in cotyledons of germinating soybeans in a previous report.? During germination, in cotyledons of germinating soybean which were grown either in the dark or the light, St was predominat component and the others were minor ones. In cotyledons, ASG markedly decreased in the initial stage of germination under the two conditions, while SE increased, especially in the lightgrown seedlings.
Changes in sterol compositions of the four classes of sterol lipids in each tissue

The changes in sterol compositions of SE, St, ASG and SG in the three tissues of soybean seedlings are shown in Figs. 4, 5, 6 and 7 , respectively. During germination, the major sterol components in each tissue, which did not depend on the germinating conditions, were $\beta$-sitosterol and stigmasterol, the minor ones being campesterol and cholesterol etc. During germination, the changing patterns of the sterol compositions of ASG and SG were very similar between the dark-grown and light-grown seedlings, and among the three tissues with a few exceptions. The changing patterns of the sterol compositions of SE and St in the two tissues (epicotyl and root) were also similar between

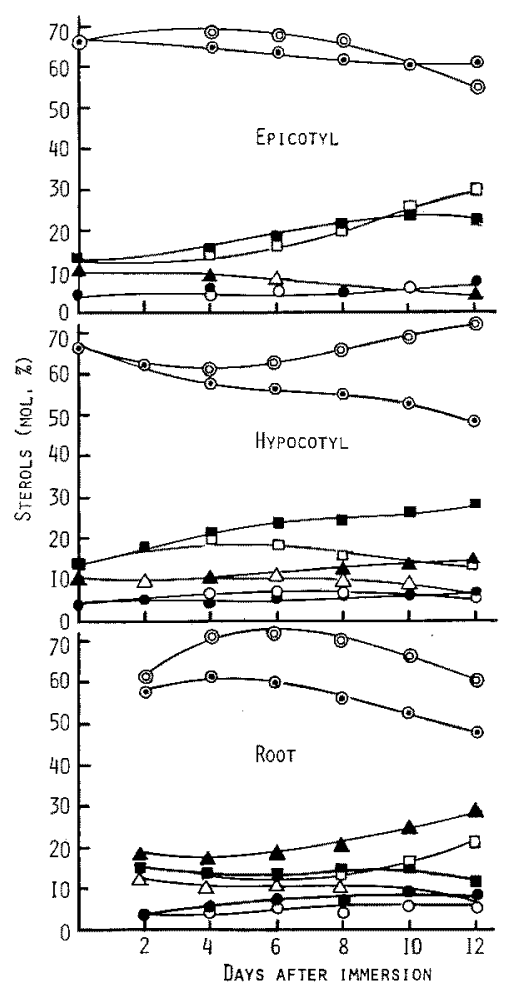

FIG. 4. Changes in Sterol Composition of Fatty Acid Esters of Sterols in Each Tissue of Soybean Seedlings during Germination.

$\beta$-Sitosterol: $\odot$, dark; $\odot$, light. Stigmasterol: dark; $\square$, light. Campesterol: $\Lambda$, dark; $\Lambda$, light. Others (cholesterol, triterpene alcohol, etc.): dark; 0 , light. 
the dark-grown and light-grown seedlings, whereas a little difference in those of hypocotyl was observed under the two conditions tested. No remarkable difference in the changing patterns of each sterol in the three tissues was observed between ASG and SG.

The percentage of $\beta$-sitosterol in ASG and $S G$ decreased in epicotyl and hypocotyl, whereas that of stigmasterol markedly increased in the two tissues. The percentages of campesterol, chloesterol etc. of ASG and $\mathrm{SG}$ in each tissue remained at almost constant values under the two conditions tested. The compositions and changing patterns of the sterol in the three tissues were more marked in St than in SE. The percentage of $\beta$-sitosterol of SE and $\mathrm{St}$ in hypocotyl markedly decreased in the dark-grown seedlings, while that in the light-grown ones decreased and then markedly increased. However, the

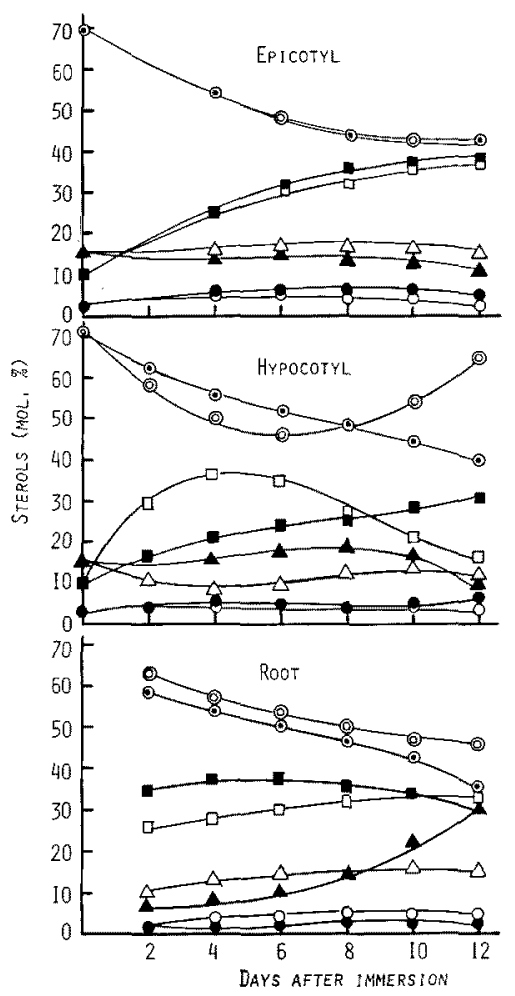

FIG. 5. Changes in Sterol Composition of Free Sterols in Each Tissue of Soybean Seedlings during Germination.

The symbols are the same as in Fig. 4.

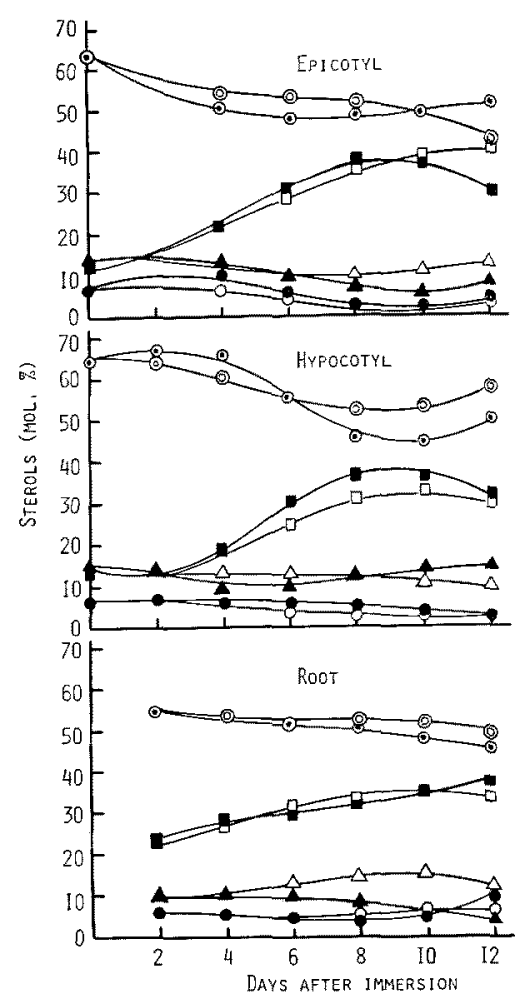

Fig. 6. Changes in Sterol Composition of Acylated Steryl Glycosides in Each Tissue of Soybean Seedlings during Germination.

The symbols are the same as in Fig. 4.

percentage of stigmasterol of SE and St in this tissue markedly increased in the darkgrown seedlings, while that in the light-grown ones increased and then decreased. A difference in the changing patterns of each sterol in root was observed between SE and St. Under the two conditions, the percentage of $\beta$-sitosterol markedly decreased in $\mathrm{St}$, whereas that in SE slightly increased and then decreased. The changes in stigmasterol of $\mathrm{St}$ in root differed from those of the other sterol lipids.

\section{Changes in fatty acid compositions of $S E$ and $A S G$}

The changes in the fatty acid compositions of SE and ASG during germination are shown in Tables I and II, respectively. SE was found to have high proportions of unsaturated fatty acids (linoleic and linolenic acids), 


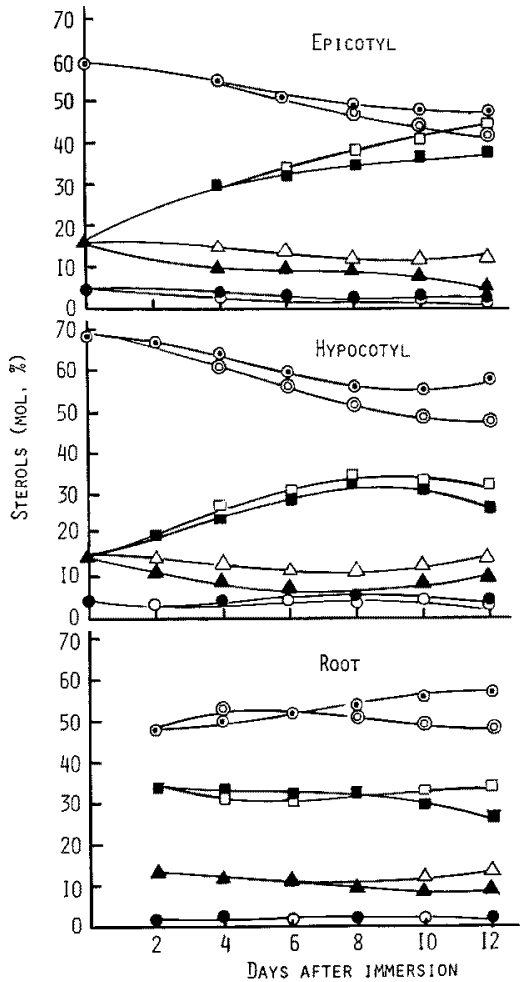

FIG. 7. Changes in Sterol Composition of Steryl Glycosides in Each Tissue of Soybean Seedlings during Germination.

The symbols are the same as in Fig. 4.

whereas ASG had high proportions of saturated fatty acids (palmitic and stearic acids), respectively. The relative amounts of linoleic acid in SE decreased with the progress of soybean seedlings. This decrease was replaced by increase of the relative amounts of linolenic acid. The changes were more marked in the light-grown seedlings than in the dark-grown ones. But the content of linolenic acid was not so much as that in cotyledons described previously. ${ }^{\text {? }}$

With the progress of germination, in the ASG of dark-grown seedlings the relative amounts of saturated fatty acid increased, accompanied with the decrease in that of linoleic or linolenic acid. However, in ASG of light-grown seedlings the relative amounts of saturated fatty acid decreased, with the increase in those of oleic and linoleic or linolenic acid. Under the two germinating conditions, saturated fatty acid, especially palmitic acid, were highly concentrated in ASG. The results are in good agreement with those described previously, ${ }^{7 /}$ and also similar to the observations of Eichenberger ${ }^{10}$ and Lepage ${ }^{11)}$ using barley, leek and potato tubers.

Changes in sugar components of $A S G$ and $S G$

The changes in the sugar components of ASG and SG during germination are shown in Fig. 8. During germination, the major sugar component of ASG and SG in epicotyl was glucose, and the minor ones were galactose and others. The sugar components in hypocotyl and root were similar to those of the epicotyl (data not shown). The percentage of glucose in ASG decreased immediately on the 4 th day after immersion, especially in the light-grown epicotyl and hypocotyl, whereas that of galactose increased in the same stage. During this period, the axis showed a marked growth and greenness of epicotyl (containing young leaves). An increase of galactose may be due to the presence of chlo-
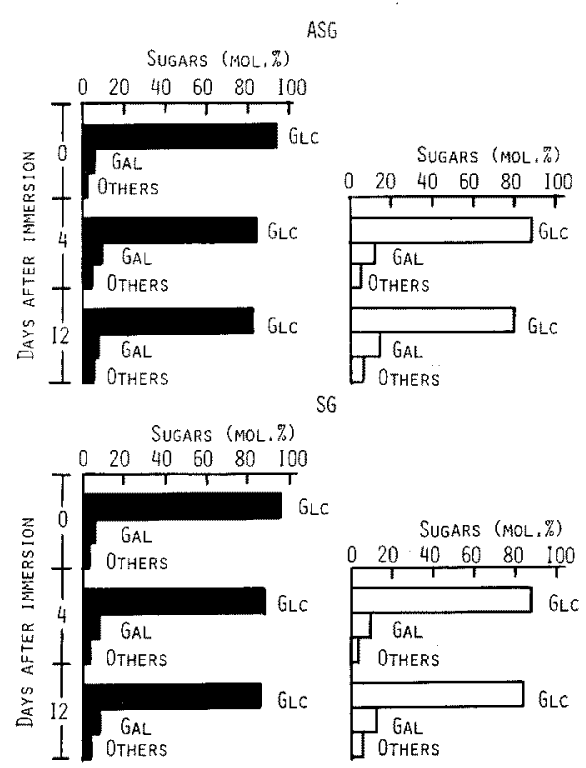

FIG. 8. Changes in Sugar Components of Acylated Steryl Glycosides and Steryl Glycosides in Epicotyl during Germination.

Glc, glucose; GAL, galactose; Others, unknown. $\mathbf{v}$, dark; $\square$ light. 


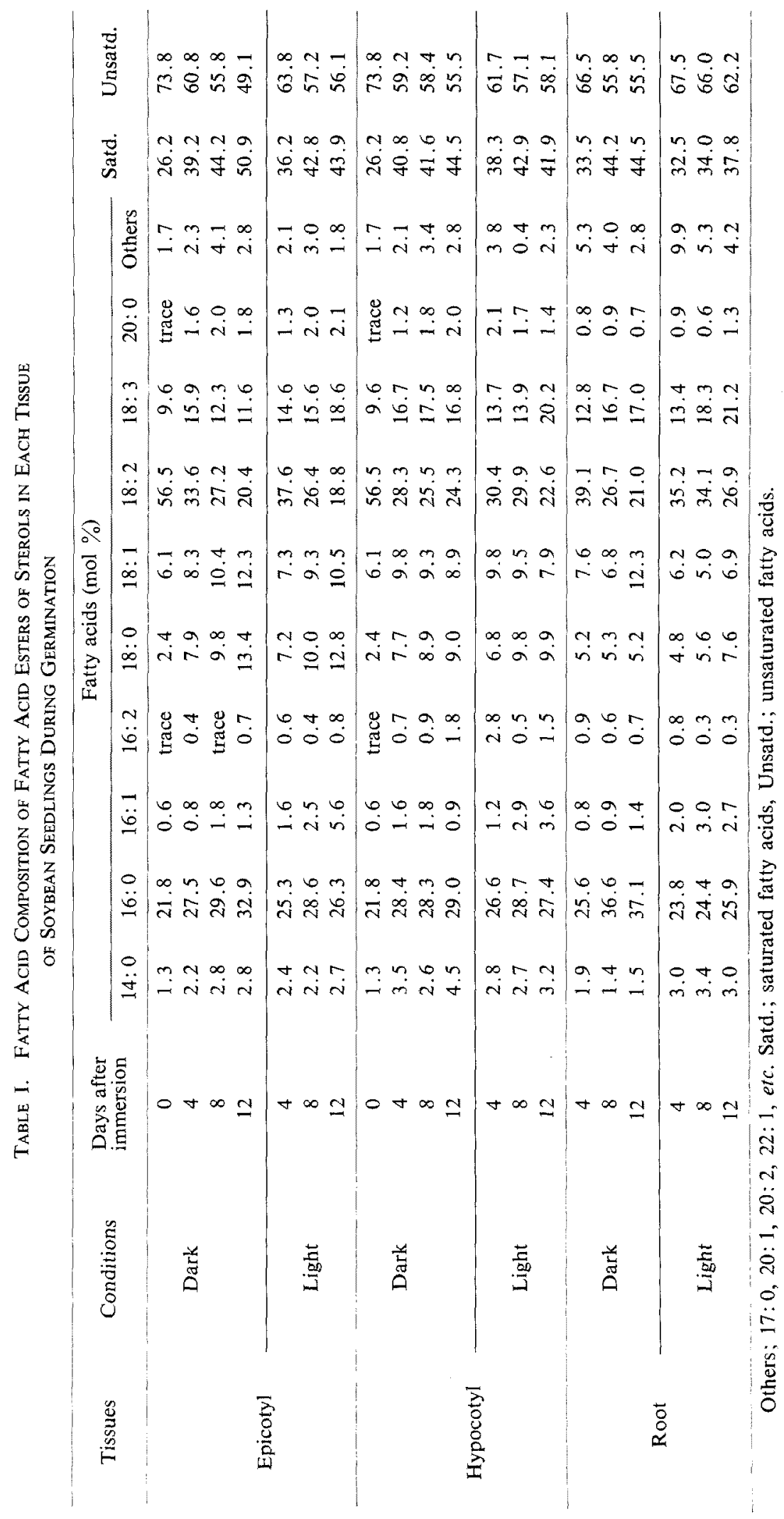




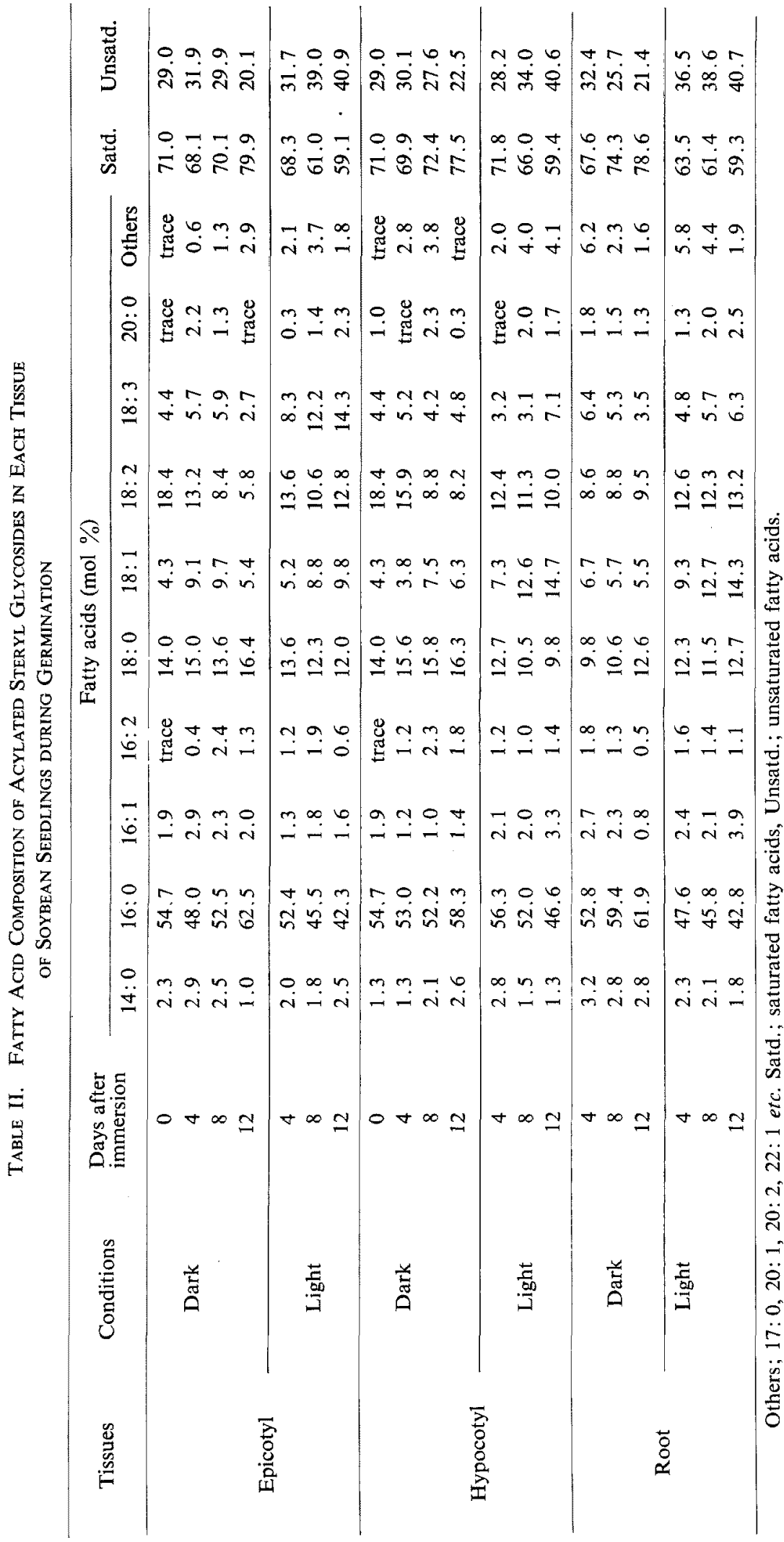


roplasts and glycolipids in it. During germination, no remarkable difference in the changing patterns of the sugar components of ASG and SG was observed between the dark-grown and light-grown seedlings.

\section{DISCUSSION}

In axis and root of germinating soybeans which were grown either in the dark or the light, the sterol contents of four classes of sterol lipids increased in two forms (ASG and SG), and were almost constant in the other two forms ( $\mathrm{St}$ and $\mathrm{SG}$ ). The results markedly differ from those of cotyledons described previously, ${ }^{7}$ in which the sterol contents have decreased in two forms (St and ASG) and increased in the other two forms (SE and SG). The content of ASG markedly increased in epicotyl and hypocotyl of light-grown seedlings, especially on the 4 th day after immersion (Figs. 1 and 2). During germination of soybean seeds, the axis showed a marked growth of hypocotyl during zero to 3 days and of epicotyl during 4 to 12 days after immersion. Perphaps owing to the greening that occurred when soybean seedlings were grown under the light, the photosynthetic tissues developed in the light-grown seedlings, especially in epicotyl containing young leaves. The amounts of total sterol in each tissue were more marked in the light-grown seedlings than in the dark-grown ones (Figs. 1, 2 and 3). The biosynthesis of many terpene derivatives has been shown to be influenced by light. ${ }^{12 \sim 14}$ Sterols and carotenes are synthesized in different compartments within the cell: carotenes in the chloroplasts, and sterols in the cytoplasm. ${ }^{15,16)}$ Since phytochrome exerts a controlling influence on carotene synthesis, it would be of interest to determine whether the pigment also regulates sterol biosynthesis.

During germination, the major sterol components of the four classes of sterol lipids in the three tissues (epicotyl, hypocotyl and root) were $\beta$-sitosterol and stigmasterol, the minor ones being campesterol and cholesterol etc. The results somewhat differ from the patterns of sterols in plant seeds. ${ }^{17}$ ) In general, with the progress of axis and root, the content of stigmasterol in sterol lipids was higher than that of campesterol. During germination, the changing patterns of the sterol compositions of $A S G$ and $S G$ were very similar between the dark-grown and light-grown seedlings, and among the three tissues. Although very little is known about the metabolism of ASG and SG, the results obtained might reflect a metabolic relationship of them. ${ }^{18)}$ The changing patterns of the sterol compositions of SE and $\mathrm{St}$ in the two tissues (epicotyl and root) were also similar between the dark-grown and light-grown seedlings, whereas a little difference in those of hypocotyl was observed in the two conditions. The changes in each sterol of $\mathrm{St}$ were the most marked than those of the other sterol lipids.

Little is known about the physiologic function of sterols. The present state of knowledge has recently been reviewed. ${ }^{19,20}$, If plant sterols have a function similar to that of sterol in animal cells, the following roles have to be considered: constituents of cellular membranes and hormones or precursors thereof. In general, sterols are rich distributed in the embryo of seeds with terpene derivatives and the ratios of them increase during germination. ${ }^{21}$ It is of interest that the increases of total sterols were observed in the three tissues as shown in Figs. 1, 2 and 3.

The data on the fatty acid compositions in the three tissues showed that SE was found to have high proportions of unsaturated fatty acids (linoleic and linolenic acids), whereas ASG had high proportions of saturated fatty acids (palmitic and stearic acids). As for the fatty acid compositions during germanation, a distinct difference was observed between the dark-grown and light-grown seedlings. The content of palmitic acid in SE and ASG of the dark-grown seedlings was high, and that of linoleic or linolenic acid was low in comparison with those of the light-grown 
ones. The results suggest the difference of the capacity for both sterol lipid synthesis and fatty acid desaturation of soybean seedlings, probably owing to that in the two germinating conditions and are also consistent with the observations by Negishi ${ }^{22)}$ and Murphy ${ }^{23)}$ using soybean seedlings and cucumber cotyledons, respectively.

No remarkable difference in the compositions and changing patterns of sugars in ASG and SG was observed between the dark-grown and light-grown seedlings, and among the three tissues, respectively. Generally, the sugar part of ASG and SG in plant cell consists mostly of glucose. ${ }^{11,24)}$ We need to investigate in datail the occurrence of galactose when soybean seedlings are grown under the light. This evidence on sterols and sugars of sterol lipids would suggest that SG may be a metabolic intermediate in the biosynthesis of ASG from sugar. ${ }^{18)}$

\section{REFERENCES}

1) S. F. Fleischer and H. Klouwen, Biochem. Biophys. Res. Commun., 5, 378 (1961).

2) L. L. van Deenen, J. Am. Oil Chem. Soc., 43, 296 (1966).

3) R. J. Kemp and E. J. Mercer, Biochem. J., 110, 119 (1968).

4) G. Hømer, R. L. Ory and C. -E. Høy, Lipids, 8, 277 (1973).

5) M. Katayama and M. Katoh, Nippon Nögeikagaku Kaishi, 48, 221 (1974).
6) H. Yoshida and G. Kajimoto, Agric. Biol. Chem., 41, 2431 (1977).

7) H. Yoshida and G, Kajimoto, ibid., 43, 705 (1979).

8) J. Folch, M. Lees and G. H. Sloane-Stanley, J. Biol. Chem., 226, 497 (1957).

9) W. Stoffel, F. Chu and E. H. Ahrens, Anal. Chem., 31, 307 (1959).

10) W. Eichenberger and E. C. Grab, FEBS Lett., 20, 245 (1972).

11) M. Lepage, J. Lipid Res., 5, 587 (1964).

12) P. Dupèron and R. Dupéron, CR Hebdomad Sci., 261, 3459 (1965).

13) G. M. Jacobsohn and M. J. Fry, Arch. Biochem. Biophys., 127, 655 (1968).

14) D. R. Threllfall and W. T. Griffiths, in "Biochemistry of Chloroplasts," ed. by T. W. Goodwin, vol. 2, Academic Press, London, 1967, p. 373.

15) T. W. Goodwin, in "Biosynthetic Pathways in Higher Plants," ed. by J. B. Pridham and T. Swain, Academic Press, New York, 1965, p. 57.

16) L. J. Rogers, S. P. J. Shan and T. W. Goodwin, Photosynthetica, 2, 184 (1968).

17) M. Noda, Yukagaku, 20, 708 (1971).

18) A. Ongun and J. B. Mudd, Plant Physiol., 45, 255 (1970).

19) C. Grunwald, Ann. Rev. Plant Physiol., 26, 209 (1975).

20) E. Heftmann, Lipids, 6, 128 (1971).

21) E. Fedeli, G. Favini, F. Camurati and G. Jacini, J. Am. Oil Chem. Soc., 43, 254 (1966), 45, 676 (1968).

22) T. Negishi, J. Am. Oil Chem. Soc., 53, 77 (1976).

23) D. J. Murphy and P. K. Stumpf, Plant Physiol., 63, 328 (1979).

24) T. Kiribuchi, T. Mizunaga and S. Funahashi, Agric. Biol. Chem., 30, 770 (1966). 